\title{
Neonatologist-performed point-of-care functional echocardiography in the neonatal intensive care unit
}

\author{
Woei Bing $\underline{\text { Poon }}^{1}$, MrCPCH, FAMS, Keng Yean Wong ${ }^{2}$, MMed, FAMS
}

\begin{abstract}
Functional echocardiography ( $\mathrm{fECHO}$ ) refers to a bedside, limited assessment of the ductus arteriosus, myocardial performance and pulmonary or systemic haemodynamics that is brief in nature and addresses a specific clinical question or management dilemma. This point-of-care ultrasonography is increasingly used internationally and locally among neonatal units to assist with management of neonatal haemodynamic conditions. This article intends to explain the modality, its indications, interpretation and implications for management, and how it impacts long-term outcomes, particularly in chronic lung disease for premature infants born before 32 weeks of gestation. This review will focus on $\mathrm{fECHO}$ as a clinical tool to assess the haemodynamics of sick neonates and how it assists in the logical choice for cardiovascular support. Training should be approached as a combined effort between the paediatric cardiology service and neonatology service.
\end{abstract}

Keywords: functional echocardiography, neonatal haemodynamics, patent ductus arteriosus, point-of-care ultrasonography, pulmonary hypertension

\section{INTRODUCTION}

Functional echocardiography ( $\mathrm{fECHO}$ ) refers to a bedside, limited assessment of the ductus arteriosus (DA), myocardial performance, and pulmonary or systemic haemodynamics; it is brief in nature and addresses a specific clinical question or management dilemma. It incorporates the assessment of intra- and extracardiac shunts, and can provide real-time information on the haemodynamic mechanism for circulatory failure, hence allowing the use of targeted therapy. ${ }^{(1)} \mathrm{fECHO}$, which is also known as targeted neonatal echocardiography, is performed by neonatologists and involves the focused use of echo mainly for functional assessment. In contrast, paediatric cardiologistperformed echocardiography focuses on the provision of consultative, cross-sectional assessments of structural heart disease. Although the paediatric cardiologist performs $\mathrm{fECHO}$ as well, the main difference is that in paediatric cardiologistperformed echocardiography, the neonatologist is dependent on the cardiologist to interpret structural heart disease, but the cardiologist may not be immediately present when the haemodynamics change. In $\mathrm{fECHO}$, the assumption is that the neonatologist who does the functional echocardiogram is readily available at the patient's bedside.

The importance of such terminology cannot be overemphasised, particularly in a complex environment such as the neonatal intensive care unit (NICU) where information is conveyed by different medical and multidisciplinary teams over long periods, as the two approaches have different indications and implications.

\section{Point-of-care ultrasonography in the NICU}

Point-of-care ultrasonography (US), which is also called clinicianperformed ultrasonography and denotes the use of US by clinicians at the bedside, has been increasingly utilised across diverse medical specialities in recent years. $\mathrm{fECHO}$ is an essential part of point-of-care US, helping to improve clinical assessment, but not replacing it. Clinicians can be trained to provide imaging and measurements with a specific clinical question in mind. This approach complements, rather than replaces, detailed structural assessments by consultative services such as cardiology or radiology. It can help to support clinical judgment, facilitate a better understanding of physiology, and assist in making rational management decisions and monitoring responses to treatment. Such an approach has been shown to improve clinical diagnosis and management. ${ }^{(2)}$

The advantages of $\mathrm{fECHO}$, including the provision of real-time information on haemodynamics, noninvasiveness, rapidity of data acquisition, report generation and allowing for serial functional assessments, have resulted in its increased use by neonatologists in the NICU. $\mathrm{fECHO}$ also addresses the limitations of clinical assessment in cardiovascular monitoring and can more accurately assess systemic blood flow. In comparison, clinical signs, including blood pressure, heart rate and capillary refill, give limited information regarding the adequacy of systemic blood flow and organ perfusion. ${ }^{(3)}$ Additional challenges of clinical assessment include the complexity and dynamic nature of the transitional circulation, the variable responsiveness of the immature myocardium to therapy during the early neonatal period, and the presence of intra- and extracardiac shunts, such as those from a patent ductus arteriosus (PDA).

\section{Impact of fECHO on clinical outcomes}

Current literature supports the success of using $\mathrm{fECHO}$ in the $\mathrm{NICU}$ to detect haemodynamic compromise, change patient management and improve outcomes. ${ }^{(4)}$ For instance, the introduction of a neonatologist-performed screening programme for haemodynamically significant ductus arteriosus (HSDA) on

${ }^{1}$ Department of Neonatal and Developmental Medicine, Singapore General Hospital, 2Paediatric Cardiology Pte Ltd, Singapore

Correspondence: Dr Poon Woei Bing, Senior Consultant Paediatrician and Neonatologist, Department of Neonatal and Developmental Medicine, Singapore General Hospital, Outram Road, Singapore 169608. poon.woei.bing@sgh.com.sg 
Day 3 of life with targeted intervention was associated with a temporal reduction in severe intraventricular haemorrhage and ventilation duration. ${ }^{(5)}$ A retrospective review of $\mathrm{fECHO}$ in a tertiary neonatal unit, performed by neonatologists under cardiologist supervision, showed that the programme resulted in a $66 \%$ rate of change in direct management. ${ }^{(6)}$ Lee et al showed that $\mathrm{fECHO}$ can provide quick and accurate identification of PDA with $87 \%$ sensitivity and $71 \%$ specificity, ${ }^{(7)}$ while Moss et al reported a $82 \%$ concordance between imaging by trained neonatologists and those by paediatric cardiologists. ${ }^{(8)}$

\section{US techniques}

For diagnostic accuracy, it is important to have quality images produced by competent operators. Serial examination of the patient should ideally be performed by one examiner to limit interobserver variability, assuming that the examiner is reliable (i.e. intraobserver variability). However, it can also be argued that having several trained observers is more pragmatic, as long as they are equally well-trained. $\mathrm{fECHO}$ includes evaluation using two-dimensional methods, pulsed wave Doppler, continuous wave Doppler and M-mode methods. Common windows used include the apical, long axis and short axis parasternal, subcostal, ductal and suprasternal views.

Image quality may be compromised by hyperinflated lungs, particularly in neonates with bronchopulmonary dysplasia. Furthermore, excessive handling or chest compression from overzealous probe positioning may lead to decompensation in preterm infants. The duration of studies should be limited, balancing the need for information against patient stability. Excellent resolution with adequate tissue penetration can usually be obtained in term and preterm infants using a probe frequency of $7.5-10 \mathrm{MHz}$.

\section{Clinical applications of fECHO}

The following clinical situations are indications for performing a fECHO study: (a) myocardial function assessment, hypovolaemia and organ perfusion; (b) PDA haemodynamic assessment and clinical relevance; (c) assessment of pulmonary haemodynamics in pulmonary hypertension; and (d) other situations such as line placement, perinatal asphyxia and post-extensive resuscitation.

\section{Myocardial function assessment, hypovolaemia and organ perfusion}

Approximately one-third of preterm neonates have systemic hypotension and up to $40 \%$ require vasopressor therapy. ${ }^{(9)}$ Overreliance on blood pressure, in isolation, as the sole marker of the adequacy of systemic blood flow is problematic. First, the arbitrary threshold of mean arterial pressure approximating the gestational age, which was proposed in 1992, lacks scientific validation. Second, end-organ perfusion is dependent on both systemic blood flow and vascular resistance; therefore, blood pressure does not provide adequate information on organ perfusion. This is best illustrated by Kluckow and Evans' study, which demonstrated a poor correlation between blood pressure and left ventricular output (LVO) on the first day of life. ${ }^{(10)}$ Furthermore, a poor correlation has been found between blood pressure and superior vena cava flow representing upper body circulation, including the brain. ${ }^{(11)}$ Both blood pressure and systemic blood flow are important as determinants of altered perfusion and should not be assessed in isolation. Serial echocardiography offers insights into haemodynamic impairment; specifically, on whether it relates to preload, afterload or myocardial contractility.

Doppler assessments of LVO and right ventricular output (RVO) provide additional information about the adequacy of systemic blood flow. LVO requires careful interpretation, as it may be falsely reassuring in the context of PDA. Early post-PDA ligation LVO can predict late-onset impairment in left ventricular (LV) contractility, low systolic blood pressure and the need for cardiotropes. ${ }^{(12)}$ Impaired LV contractility following PDA ligation is due to hypertension or increased systemic afterload following ductal closure. The depressed ventricular function results in blood pressure that is near normal or appears low, and inotropes may worsen it. Treatment involves vasodilator drugs such as glyceryl trinitrate, milrinone or a low-dose diuretic. Hence, it has been proposed that RVO and superior vena cava flow are less confounded by PDA, particularly in the context of the transitional circuit. ${ }^{(13)}$

Evaluation of myocardial contractility is challenging, as current methods are load-dependent, although serial measurements provide valuable insights. The immature neonate is especially sensitive to sudden changes in afterload, specifically during the transition period after elimination of the placenta from the systemic circulation, and following PDA ligation. LV systolic performance can be assessed using the shortening fraction (SF), ejection fraction or rate-corrected mean velocity of circumferential fibre shortening. Normal values for neonatal SF are $28 \%-40 \%$. However, SF values must be interpreted cautiously in the first few days of life, because high right ventricular (RV) pressures may impair ventricular septal movement. ${ }^{(14)}$

By understanding systemic blood flow, hypotension and/or low blood flow can be logically managed using cardiovascular medications, with respect to their known physiologic effects on contractility and vascular resistance. Hypotension with low systemic blood flow suggests a high systemic vascular resistance, and this might benefit most from increased contractility and afterload reduction with medications such as dobutamine and milrinone. In hypotension with normal systemic blood flow, dopamine can be used to increase afterload and blood pressure. ${ }^{(13)}$ A large unrestrictive PDA can contribute to early hypotension in very-low-birth-weight infants. A low cardiac output state may be caused by or be a result of metabolic acidosis. Correct interpretation of echocardiographic findings is important in deciding what measures should be given.

When evaluating for the presence of neonatal hypotension, ${ }^{(15)}$ essential steps are the assessment of: (a) systemic blood flow using LVO as well as RVO; (b) structural heart disease; (c) LV function, including LV systolic function using ejection fraction and SF; (d) pulmonary hypertension; and (e) ductal patency. 


\section{PDA haemodynamic assessment and clinical relevance}

Controversy remains about the definition of HSDA, the role of PDA in adverse outcomes, and the most appropriate mode and timing of treatment. PDA is dynamic, with variable architecture and unpredictable treatment response. The magnitude of transductal flow is difficult to quantify, although its impact on pulmonary and systemic circulations is more easily quantified.

There are several approaches to medical management: prophylactic treatment (within 12 hours after birth), early targeted treatment (6-72 hours after birth), treatment when clinical signs of PDA appear and conservative management. A systematic review of prophylactic treatment showed a reduction in symptomatic DAs (relative risk [RR] 0.44, 95\% confidence interval $[\mathrm{Cl}]$ 0.38-0.50), but no difference in longterm neurodevelopmental outcome despite a lower incidence of intraventricular haemorrhage. ${ }^{(16)}$ It therefore seems that the infants whose PDAs would otherwise close spontaneously were unnecessarily exposed to indomethacin during prophylactic treatment, and do not benefit from it or may even suffer adverse effects. With the prophylactic approach, approximately $40 \%$ of patients were unnecessarily treated. ${ }^{(13)}$

Early targeted treatment selects the treatment based on $\mathrm{fECHO}$ parameters, which predict to some extent the likelihood of DA persisting and requiring treatment; however, multiple trials have not shown any benefits so far. ${ }^{(17)}$

Treatment after the appearance of clinical signs avoids overtreatment, but clinical signs such as murmur and wide pulse pressure appear late in the clinical course, and significant ductal steal reducing organ perfusion may occur prior to clinical signs. This is a widely practised approach that has not been subjected to rigorous trials. Although the definition of HSDA is still controversial, a pragmatic approach that has been proposed is to gather as much information as possible on the duct using $\mathrm{fECHO}$, then quantify the DA into small, moderate or large using clinical judgment. Suggestions for the clinical classification of PDA using clinical and $\mathrm{fECHO}$ markers have been proposed in the literature; in particular, McNamara and Sehgal have designed a clinical and fECHO staging system, which could be used to define thresholds for pharmacological treatment. ${ }^{(18,19)}$ Less aggressive and more permissive management for PDA may not result in worse outcomes. In some retrospective cohort studies, management strategies that avoid the use of indomethacin or ibuprofen had outcomes similar to those of medical treatment. ${ }^{(20)}$

In summary, more research is required on the selective use of medical methods of ductal closure, either for high-risk infants in the first two weeks of life or for older infants with persistent PDA. Additional research needs to address, first, the relationship between haemodynamic significance and increased risks of prolonged patency and adverse clinical outcomes, such as chronic lung disease (CLD) or neurodevelopmental impairment. Second, well-designed and-executed intervention trials are needed, with clinically important long-term outcomes as the endpoints, and not simply rates of ductal closure or measures of short-term physiologic changes. ${ }^{(20)}$

\section{Assessment of pulmonary haemodynamics in pulmonary hypertension}

Persistent pulmonary hypertension of the newborn (PPHN) is a diagnosis made with echocardiography; this modality also provides confirmation of normal cardiac anatomy. As time-critical therapeutic decisions are often required in cases of PPHN, $\mathrm{fECHO}$ helps to rule out major anatomical abnormalities and establish pulmonary pressures. ${ }^{(21)}$

On the other spectrum, preterm infants with CLD and oxygen requirement may have subclinical pulmonary hypertension. Evaluation of such neonates may include evaluation of pulmonary pressures; RV performance and pulmonary haemodynamics; and LV performance and systemic blood flow. Right ventricular systolic pressure (RVSP) can be measured in the presence of tricuspid regurgitation and calculated using the Bernoulli equation: RVSP $=$ right atrial pressure $+\left(4 \times v_{2}\right)$, where $v^{2}$ : tricuspid regurgitation jet peak velocity.

However, pulmonary pressures may be underestimated if there is impaired RV contractility. The presence of PDA may also be used to estimate pulmonary pressures if systemic systolic pressures are known.

Transductal flow direction can also be used to estimate the severity of pulmonary hypertension. A pure unrestrictive rightto-left shunt suggests suprasystemic pulmonary hypertension, while a bidirectional shunt suggests pulmonary pressure that approximates systemic pressure. If these shunts are absent, direct assessment of pulmonary pressures is difficult, particularly in those with CLD. The pulmonary artery ejection time to RV

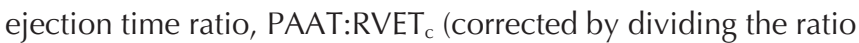
with RR interval on simultaneous electrocardiography), derived from pulmonary artery Doppler, has been shown to negatively correlate with pulmonary pressures in premature infants and is a good predictor of late-onset CLD. ${ }^{(22)}$

Increased RV afterload from pulmonary hypertension may impair RV contractility. Although moderate-to-severe RV systolic dysfunction is usually obvious via direct visualisation, there is no reliable marker of RV failure. In RV dysfunction, the RV appears larger and stiffer compared to the LV, with dilated RV outflow tract, and contractility may appear subjectively diminished. The interventricular septum may be flattened, or even show paradoxical septal motion in severe cases, which manifests as reversed septal motion; these may affect the accuracy of indices of LV function on M-mode echocardiography. Low RV output $(<170 \mathrm{~mL} / \mathrm{kg} / \mathrm{min})$ may also support the pulmonary hypertension diagnosis and could be useful in monitoring the patient's response to inodilators such as milrinone. Understanding the physiology of PPHN in terms of the presence or absence of LV dysfunction helps in selecting appropriate drugs; inhaled nitric oxide (iNO) and oral medications such as milrinone or vasopressin may not be needed in every case. ${ }^{(13)}$

$\mathrm{fECHO}$ has the following roles in PPHN:(15)

- $\quad$ Confirmation and quantification of PPHN severity using tricuspid regurgitant jet, ductal shunt direction, septal wall motion and PAAT:RVET ratio;

- Exclusion of contraindications to iNO, which include 
congenital heart disease characterised by ductal-dependent systemic blood flow (e.g. interrupted aortic arch, critical aortic stenosis, hypoplastic left heart syndrome) and severe LV dysfunction;

- Evaluation of RV performance using RV contractility and output;

- Evaluation of LV performance using LV diastolic performance, contractility and output; and

- Allowing the use of physiology-based targeted medications, i.e. treating low preload states with volume resuscitation, reduction of ventilator mean airway pressure or the use of iNO, treating reduced LV or RV contractility with dobutamine and/or milrinone, and considering the usage of vasopressin in patients with both low LV or RV contractility and systemic vasodilatation.

\section{Other less common indications}

Central line placement and perinatal asphyxia are less common indications for the use of $\mathrm{fECHO} .{ }^{(15)}$ On $\mathrm{fECHO}$ subcostal parasagittal views, the inferior vena cava-right atrial junction can be easily seen, and may facilitate checking of umbilical venous catheter, umbilical arterial catheter and central venous line placement. Infants with perinatal asphyxia represent another patient subgroup that may benefit from $\mathrm{fECHO}$. Transient myocardial ischaemia may be present in these infants, with impaired LV function, mitral and/or tricuspid regurgitation, LV dilation, LV wall-motion abnormalities and decreased cardiac output. Those with hypotension may benefit from the use of dobutamine for afterload reduction and increased contractility. Careful fluid adjustment with either fluid restriction or volume expansion can be guided according to the end-diastolic volume of the LV, chamber filling and inferior vena cava status, which are all reduced in hypovolaemia.

\section{Prognosticating long-term outcomes}

A ductal staging system has been developed by Sehgal et al that may help to predict the development of CLD, based on a preterm infant cohort of less than 32 weeks' gestation who had HSDA requiring medical treatment. ${ }^{(23)}$ This composite scoring system consists of nine parameters (scored 0-3) that can be subdivided into three categories: ductal features, magnitude of the ductal shunt and features of myocardial performance. For every single point of increase in the composite score, the odds of CLD increased by $78 \%$ (odds ratio $1.78,95 \% \mathrm{Cl} 1.35-2.34$ ). In addition, a cut-off value of 21 and above showed a sensitivity of $88.9 \%$ and specificity of $88 \%$ for CLD. ${ }^{(23)}$

\section{Training and standards of practice}

Collaboration with paediatric cardiology is essential in setting standards of practice and to ensure that competence is achieved and maintained. The main concern regarding the use of functional US is the possibility of misdiagnosis. Protocols for the use of $\mathrm{fECHO}$ must be instituted, including performing a cardiologist assessment beforehand or, at least, shortly after the first fECHO. Reassuringly, the literature has shown that a structural cardiac abnormality is almost always detected, even if a full diagnosis may not be made. ${ }^{(22)}$ Neonatologists' studies serve a different purpose and should complement the cardiologists' assessment. Standardised training and accreditation are necessary to avoid misuse of this skill, and a combined approach between the paediatric cardiology and neonatology services is necessary.

\section{CONCLUSION}

Neonatologist-performed $\mathrm{fECHO}$ is increasingly being recognised as a useful technique and gaining momentum globally. Its impact is greatest when the echocardiography evaluation is of high quality, comprehensive and follows careful consideration of the clinical context. Standardised training and accreditation are necessary to ensure that standards of practice are maintained.

\section{REFERENCES}

1. Kluckow M, Seri I, Evans N. Functional echocardiography: an emerging clinical tool for the neonatologist. J Pediatr 2007; 150:125-30.

2. Beaulieu Y. Bedside echocardiography in the assessment of the critically ill. Crit Care Med 2007; 35(5 Suppl):S235-49.

3. Osborn DA, Evans N, Kluckow M. Clinical detection of low upper body blood flow in very premature infants using blood pressure, capillary refill time and central-peripheral temperature difference. Arch Dis Fetal Neonatal Ed 2004; 89:F168-73.

4. Seghal A, McNamara PJ. Does point-of-care functional echocardiography enhance cardiovascular care in the NICU? J Perinatol 2008; 28:729-35.

5. O'Rourke DJ, El-Khuffash A, Moody C, Walsh K, Molloy EJ. Patent ductus arteriosus evaluation by serial echocardiography in preterm infants. Acta Paediatr 2008; 97:574-8.

6. Kadivar M, Kiani A, Kocharian A, et al. Echocardiography and management of sick neonates in the intensive care unit. Congenit Heart Dis 2008; 3:325-9.

7. Lee HC, Silverman N, Hintz SR. Diagnosis of patent ductus arteriosus by a neonatologist with a compact, portable ultrasound machine. J Perinatol 2007; 27:291-6.

8. Moss S, Kitchiner DJ, Yoxall CW, Subhedar NV. Evaluation of echocardiography on the neonatal unit. Arch Dis Child Fetal Neonatal Ed 2003; 88:F287-9; discussion F290-1.

9. Al-Aweel I, Pursley DM, Rubin LP, et al. Variations in prevalence of hypotension, hypertension, and vasopressor use in NICUs. J Perinatol 2001; 21:272-8.

10. Kluckow M, Evans N. Relationship between blood pressure and cardiac output in preterm infants requiring mechanical ventilation. J Pediatr 1996; 129:506-12.

11. Kluckow M, Evans N. Low superior vena cava flow and intraventricular haemorrhage in preterm infants. Arch Dis Child Fetal Neonatal Ed 2000; 82:F188-94.

12. Sahni M, Sehgal A, Stewart L, Shivananda S, McNamara PJ. Early functional echocardiography ( $\mathrm{fECHO}$ ) predicts postoperative cardiorespiratory instability after patent ductus arteriosus (PDA) ligation. E-PAS; 2009.

13. de Waal K, Kluckow M. Functional echocardiography; from physiology to treatment. Early Hum Dev 2010; 86:149-54.

14. Gill $A B$, Weindling AM. Echocardiographic assessment of cardiac function in shocked very low birthweight infants. Arch Dis Child 1993; 68(1 Spec No):17-21.

15. Mertens L, Seri I, Marek J, et al; Writing Group of the American Society of Echocardiography (ASE); European Association of Echocardiography (EAE); Association for European Pediatric Cardiologists (AEPC).Targeted neonatal echocardiography in the neonatal intensive care unit: practice guidelines and recommendations for training. Eur J Echocardiogr 2011; 12:715-36.

16. Fowlie PW, Davis PG, McGuire W. Prophylactic intravenous indomethacin for preventing mortality and morbidity in preterm infants. Cochrane Database Syst Rev 2010; (7):CD000174.

17. Cooke L, Steer P, Woodgate P. Indomethacin for asymptomatic patent ductus arteriosus in preterm infants. Cochrane Database Syst Rev 2003; (2):CD003745.

18. Sehgal A, McNamara PJ. Does echocardiography facilitate determination of hemodynamic significance attributable to the ductus arteriosus? Eur J Pediatr 2009; 168:907-14

19. McNamara PJ, Sehgal A. Towards rational management of the patent ductus arteriosus: the need for disease staging. Arch Dis Child Fetal Neonatal Ed 2007; 92:F424-7.

20. Benitz WE; Committee on Fetus and Newborn, American Academy of Pediatrics. Patent Ductus Arteriosus in Preterm Infants. Pediatrics 2016; 137:e20153730.

21. El-Khuffash AF, McNamara PJ. Neonatologist-performed functional echocardiography in the neonatal intensive care unit. Semin Fetal Neonatal Med 2011; 16:50-60.

22. Subhedar NV, Hamdan AH, Ryan SW, Shaw NJ. Pulmonary artery pressure: early predictor of chronic lung disease in preterm infants. Arch Dis Child Fetal Neonatal Ed 1998; 78:F20-4.

23. Seghal A, Paul E, Menahem S. Functional echocardiography in staging for ductal disease severity: role in predicting outcomes. Eur J Pediatr 2013; 172:179-84. 